\title{
Effect of loading pulse duration on dynamic buckling of stiffened panels
}

\author{
O. Mouhat ${ }^{1}$, A. Khamlichi ${ }^{2}$ \\ ${ }^{1}$ Communications Systems and Detection Laboratory; Abdelmalek Essaadi University, Tetouan 93002, Morocco \\ ${ }^{2}$ Communications Systems and Detection Laboratory; Abdelmalek Essaadi University, Tetouan 93002, Morocco
}

\begin{abstract}
Design of stiffened panels requires evaluating their stability under various loading combinations for all possible scenarios regarding material degradation or initial geometric imperfections that could affect them. Both static and dynamic loading conditions are to be investigated for assessing the buckling strength. In this work, dynamic buckling under in-plane uniform axial compression loading having the form of a transient pulse with finite duration is evaluated through nonlinear finite element modelling. A welding induced defect that consists of an initial geometric imperfection modifying the skin plate curvature in the longitudinal direction was incorporated. The Budiansky buckling criterion was employed to predict instability under this dynamic loading. The obtained results have shown that the pulse period yields a drastic effect on the buckling strength. For the considered boundary conditions, half-sine like pulses having periods that are comparable to two times the period of the first mode of natural vibrations of the stiffened plate were found to reduce hugely the buckling strength, with the dynamic buckling load representing almost only half its static value.
\end{abstract}

\section{Introduction}

Stability of stiffened panels is a main concern in many engineering applications such as aircrafts and ships [1,2]. As modern structures are more and more designed to be of light thin-walled shape, buckling risk constitutes the main challenge to deal with in quest of increasing the strength-to-weight ratio. Stiffened panels are subjected to various loading conditions which can be either of static or dynamic character and are also subjected to various alterations that can take the form of material degradation or geometric imperfections.

Static buckling has been yet the most investigated issue for structures that are susceptible to show instability under the in-service applied loading and a relatively good understanding of this phenomenon has been reached [3]. Buckling of structures subjected to dynamical loads that are suddenly applied has not yet received the same deal of attention even if in practice this kind of loading is likely to occur with non negligible probability [4,5]. In the literature different approaches were presented by various investigators to define the dynamic buckling load. Simitses [6] classified the various concepts and methodologies used in estimating critical conditions for suddenly loaded elastic systems in two main approaches: equations of motion based methods and energy based ones. As this last approach is applicable mostly to conservative systems of low degrees of freedom, the approach based on the equations of motion $[7,8]$ is more suited for stiffened panels and is used in the following.
The equations governing the problem are solved for various values of parameters defining the loading to obtain the response of the system. The load parameter at which there exists a large change in the response is called critical. This approach has become prominent in the field of dynamic buckling and can be easily adapted to computational methods such as those using finite element codes.

As a model problem, the dynamic buckling of an impacted beam has been studied by Wooseok and Waas [9]. Unlike in the static buckling problem, the dynamic case results in localized non-uniform buckle mode shapes associated with the interactions between the in-plane and out-of-plane deformation responses.

Dynamic buckling of beams and plates subjected to axial impact was investigated by Weller et al. [10]. They performed numerical calculations to determine the dynamic load factor (DLF) of in-plane impacted beams and plates. Good agreement was observed with experimental results. The DLF was in general larger than unity, both for beams and plates. However, in the presence of certain values of relatively large initial geometric imperfections and for pulse durations of the applied load that are close to the first period of natural flexural vibrations of either the beam or the plate, the DLF was found to be smaller than unity resulting in the fact that dynamic buckling can be more severe than static buckling. This observation has been found to be valid also for axially impacted composite plates [11]. The experimental tests that these authors performed on 
laminated composite plates have shown that a DLF smaller than unity can be obtained for a given composite plate which is axially impacted with a compressive load of period close to the first period of natural lateral vibration of the plate.

From this brief literature review, one can see that buckling due to dynamical loading acting on structures cannot be always estimated by performing static analysis of the problem. It is then required to determine how a dynamical load could modify the buckling strength for the particular case of stiffened panels when they are loaded by a pulse impact compression load acting axially in the direction of stiffeners. In the present analysis a nonlinear incremental formulation using Dynamic/explicit procedure under Abaqus software package is used to study numerically dynamic buckling of a stiffened panel [12]. The buckling state is determined from the curve giving reaction resultant as function of end-shortening according to Budiansky and Roth criterion $[7,8]$. Fixing the load magnitude to be higher than that of the static buckling load, various pulse loads having the shape of a quarter-sine like time signal with different period durations were considered. These periods were chosen to be close to the characteristic time of the structure defined as the inverse of the first modal frequency of natural vibrations.

\section{Finite element modelling of dynamic buckling of stiffened panels}

The initial geometric imperfections and the geometric nonlinearities are taken into account in modelling the dynamically loaded stiffened panel by using the finite element method. A detailed description regarding the appropriate finite element formulation to be used for the numerical model of shell buckling problems can be found in [13]. In the following, the shell element S4R presented in Abaqus software package is used (Anonymous, 2010). This element has four nodes with six degrees of freedom at each node (nodal translations in $x, y$ and $z$ directions and nodal rotations about these axes).

Residual stresses developing after welding process induce distortions that have the main following effects: shrinkage in the transverse direction to the weld line, longitudinal shrinkage parallel to the weld line and rotation around the weld line. The ultimate form and magnitude of welding induced distortions depend on the actual welding parameters, the materials used, the geometric design of the panel being assembled and also on the preventive restraints applied during welding.

In order to identify the real involved distribution of initial geometric imperfection, distortion measurements are required for sufficiently representative samples of the stiffened panel under consideration. This data may then be used within finite element analysis to assess the effect of these distortions on the buckling strength. Lillemäe et al. [14] have measured the initial distortion for two assembled panels by welding and have obtained almost the same profile for both of them when considering the transverse direction. However in the longitudinal direction the distortion patterns measured by these authors were quite different for the two tested panels.

In the following imperfections resulting from welding in the transverse direction are not taken into account and the longitudinal distortion is assumed to be represented by a constant curvature that is orthogonal to the directions of stiffeners. The imperfect stiffened panel considered has the geometrical configuration shown in figure 1. This structure is generated by applying a revolution transformation of axis parallel to the small edges using a curvature radius of $23 \mathrm{~m}$ and an aperture angle of $10^{\circ}$. The obtained total length of the panel is $L=4.014 \mathrm{~m}$.

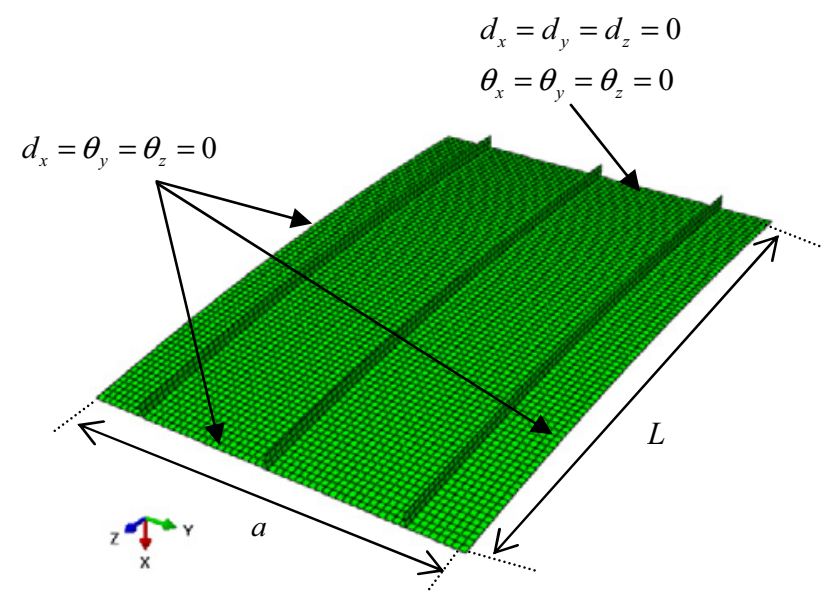

Fig. 1. Stiffened panel showing the stringers in the longitudinal direction.

The stiffened panel has three equal webs. The total width of the skin plate is $a=2.8 \mathrm{~m}$. The webs are regularly spaced with the central web placed at the transverse edge centre and parallelally to the longitudinal direction of the base plate. The two side webs are placed each at a distance $e=0.4 \mathrm{~m}$ from the nearest longitudinal edge and are also parallel to them. Their height is uniform and given by $h_{w}=0.1 \mathrm{~m}$. All the parts constituting the stiffened panel have the same constant thickness $t=3 \times 10^{-3} \mathrm{~m}$.

The material properties applied to model the stiffened panel correspond to aluminium for which the Young's modulus is $E=64.5 \mathrm{GPa}$ and Poisson coefficient is $v=0.3$.

The boundary conditions that were considered in the numerical simulations correspond to clamped transverse end edge and symmetry along the transverse direction $x$ axis for the longitudinal edges as well as for the front transverse edge. These boundary conditions are indicated in figure 1 , where the displacements are denoted $d$ and the rotations $\theta$. The dynamic in-plane compression loading is applied at the front transverse edge, it is uniform and parallel to the $z$-direction, its magnitude in $N / m$ is denoted $P_{z}$.

A finite element based modal model was developed at first. Convergence of this model was assessed with a set of 4960 SR4 elements and a total number of 30618 
degrees of freedom. The obtained first frequency of natural vibrations is $f_{0}=9.35 \mathrm{~Hz}$. Figure 2 shows the first mode of natural vibrations of the modelled structure. One can see that the first mode is essentially a flexural global skin mode.

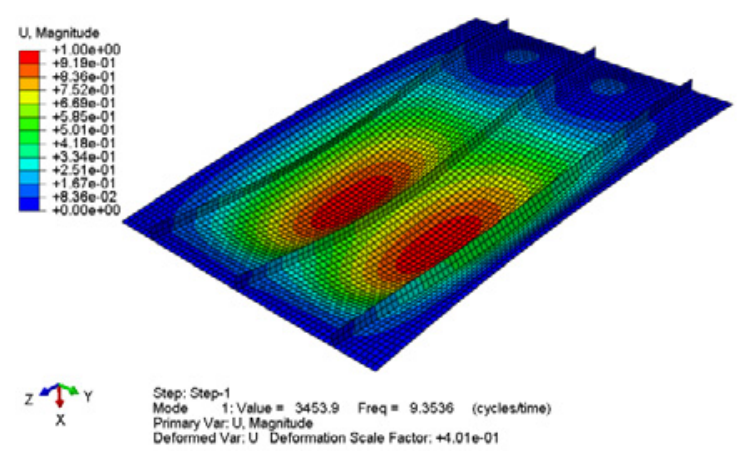

Fig. 2. First natural mode of vibrations of the stiffened panel; the associated first modal frequency is $f_{0}=9.35 \mathrm{~Hz}$.

The first modal frequency $f_{0}$ is used to compute the characteristic time $T_{0}=1 / f_{0}$. This period is used in order to fix the pulse duration for the dynamic loading to be applied to the stiffened panel by assuming for it a halfsine profile shape with a duration $T / 4$. This duration corresponds to the first increasing part of a sine like time function of period $T$. Fixing the magnitude of the load denoted $F_{0}$ at a value sufficiently large to observe dynamic buckling for all chosen durations of the applied pulse, the loading pulse is taken according to the following time function

$$
F(t)=F_{0} \sin ^{2}\left(\frac{\pi t}{T}\right), t \in\left[0, \frac{T}{2}\right]
$$

In the literature various criteria have been proposed for dynamic stability. The most widely used is however the criterion of Budiansky and Roth, where it is assumed that the instability occurs when the displacement rate is the highest for a fixed force increment. This can also be identified as the lowest load at which there is a large sudden change in the transient response. The critical value of dynamic load corresponding to loss of stability can then be found by drawing a graph of end-shortening as a function of reactions resultant.

In the following, the critical conditions for dynamic buckling are estimated according to Budiansky and Roth judgment. Some problems exist however when employing this criterion for stiffened plates which do not experience unstable post-buckling as they do not exhibit violent change in end-shortening due to dynamic buckling. The dynamic load is assumed then to be either that for which large-oscillatory motion is observed, or large end-shortening under almost constant resultant of reactions.

To compare the dynamic buckling load with the static buckling one, the dynamic load is divided by the lowest static load as computed by means of the nonlinear incremental method provided by the standard Static/Riks procedure of Abaqus software. The same model is used as in the dynamic problem regarding geometry, materials and boundary conditions. An ambiguity appears as well in defining the static buckling load because of the existence of multiple equilibrium trajectories due to multiple bifurcations that occur on the fundamental path of equilibrium and which are obtained by simply changing the initial arc length during the analysis. Figure 3 illustrates some of them.

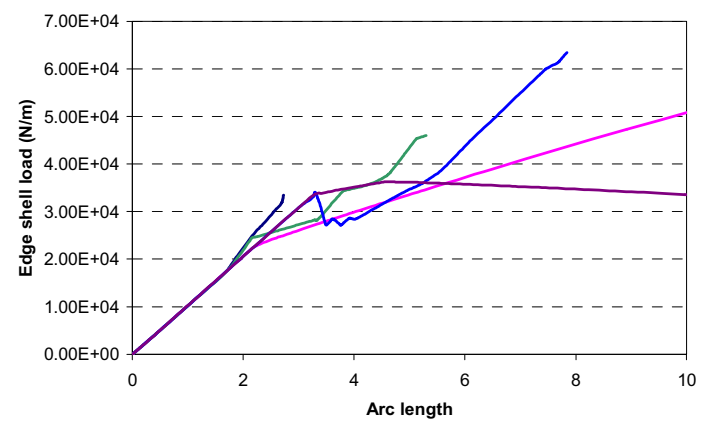

Fig. 3. Static buckling; multiple bifurcation paths as obtained by modifying the initial arc length value.

The static buckling bifurcation load is the lowest bifurcation point which corresponds in figure 4 to $P_{z, s}=20.36 \mathrm{kN} \cdot \mathrm{m}^{-1}$. But the stiffened panel can withstand uniform edge shell loading without experiencing instable post-buckling behaviour up to $P_{z, s, \lim }=33.44 \mathrm{kN} \cdot \mathrm{m}^{-1}$.

\section{Results and conclusions}

The procedure Dynamic/Explicit of Abaqus software is used with the option of automatic incrementation to solve the equations of motion, so as to obtain the dynamic responses. 20 steps were chosen for each case with a constant time step equal to $T / 40$ where $T$ is the duration of the pulse signal, so as calculation is performed only for the increasing part of the load, $t \in[0, T / 2]$. This is in order to avoid confusion with the unloading due to the applied compression is time is greater than $T / 2$.

The stiffened panel is now dynamically excited by short time duration pulse edge load corresponding to values in the following set $T \in\left\{0.5 T_{0}, T_{0}, 1.5 T_{0}, 1.9 T_{0}, 2 T_{0}, 2.1 T_{0}, 2.5 T_{0}, 5 T_{0}\right\}$.

Simulation of the response of the stiffened panel when subjected to each case of these dynamic loading was performed. The obtained results, in terms of mean reaction acting on the anchored edge as function of mean end-shortening experienced by the front edge, are presented in figure 5 . The mean reaction is obtained by summing the amplitudes of nodal reactions in the anchored edge $z=0$ and divining the result by the total number of nodes meshing this edge which is here equal to 57 . The mean end-shortening is obtained by averaging 
the displacement along the $z$ direction for the nodes located on the front edge where the pulse load is applied, that is to say $z=L$.

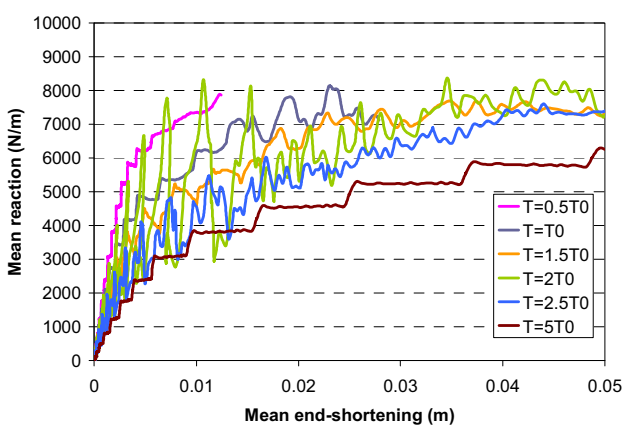

Fig. 4. Mean reaction as function of mean end-shortening for different values of pulse duration in the set $T \in\left\{0.5 T_{0}, T_{0}, 1.5 T_{0}, 2 T_{0}, 2.5 T_{0}, 5 T_{0}\right\}$.

One can notice from figure 4 that the response is apparently quasi-static for long periods of the pulse $T \geq 5 T_{0}$ as the dynamical aspect of the loading is not strong. This observation can apply also for short periods $T \leq 05 T_{0}$ as in this case too the reactions are following the applied load steps in a staircase form. For periods that are in the interval $\left[1.5 T_{0}, 2.5 T_{0}\right]$ the response is shown to be of oscillatory type and the highest amplitude of oscillations corresponds to $T=2 T_{0}$.

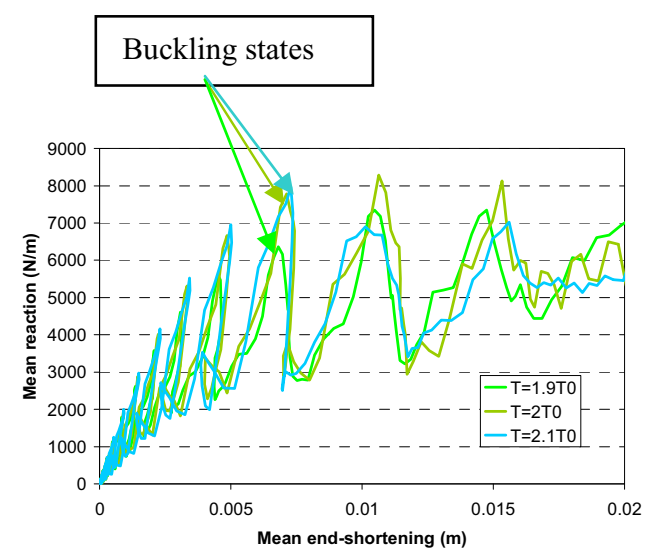

Fig. 5. Zoom in the vicinity of the origin for the curves giving mean reaction as function of mean end-shortening for pulse durations around $2 T_{0}$; the arrows indicate the buckling states.

Figure 5 gives a zoom of the oscillatory response for periods that are close to $T=2 T_{0}$, for a window limited to mean end-shortening not exceeding $0.02 \mathrm{~m}$. One can see clearly the strong oscillations indicating dynamic buckling taking place in the system. Even if the oscillations appear very early for the three periods around $T=2 T_{0}$, the buckling load can be estimated conventionally to be that one associated to the first peak which is followed by a drop of amplitude bigger than half the maximum mean reaction attained. These points are indicated in figure 5. One can now identify the applied loading corresponding to these dynamic buckling states. The obtained results are summarized in table 1 .

Table 1. Dynamic buckling load and the associated DLF as function of the pulse period.

\begin{tabular}{|c|c|c|c|}
\hline & Time $(s)$ & $\begin{array}{c}\text { Applied } \\
\text { edge load } \\
(N / m)\end{array}$ & $\begin{array}{c}\text { Dynamic load } \\
\text { factor (DLF) }\end{array}$ \\
\hline$T=1.9 T_{0}$ & 0.0644977 & 9754 & 0.48 \\
\hline$T=2 T_{0}$ & 0.0670998 & 10847 & 0.53 \\
\hline$T=2.1 T_{0}$ & 0.0698892 & 12019 & 0.59 \\
\hline
\end{tabular}

Table 1 shows that the dynamic buckling load is smaller than the static buckling one as the DLF is only about $50 \%$. A drop of about half the static load is achieved when the pulse period is around $2 T_{0}$. This duration is linked to the first mode of natural vibrations of the stiffened panel with the same boundary conditions. It should be mentioned that the conventional dynamic buckling load as defined here is larger than that for which high amplitude oscillations are observed. Instability appears in fact for all the peaks on the left of the marked peaks shown in figure 5 .

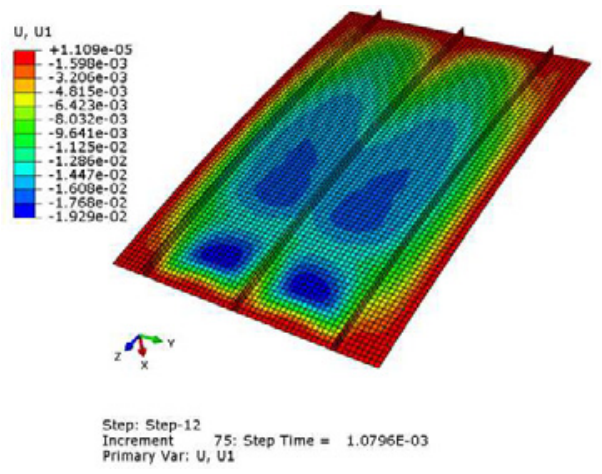

Fig. 6. Isovalues or $x$ displacement for the dynamically buckled shape of the stiffened plate with $T=2 T_{0}$.

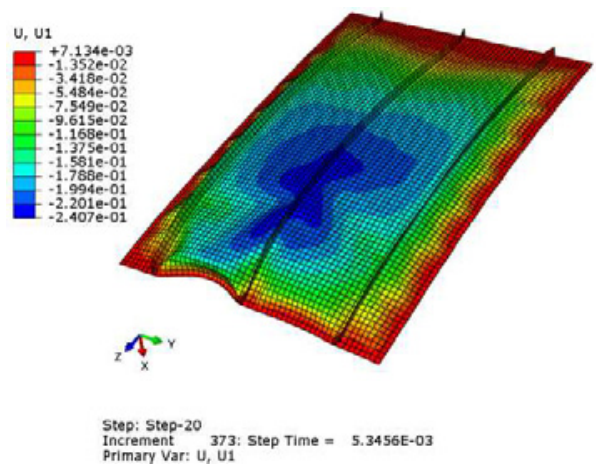

Fig. 7. Isovalues or $x$ displacement for the final collapsed shape of the stiffened plate with $T=2 T_{0}$. 
Figure 6 shows the buckled shape corresponding to the pulse period $2 T_{0}$. One can see that this buckled shape is close the first mode of natural vibrations with some localisation of deformation.

Figure 7 gives the final collapsed shape for the case corresponding to the pulse period $2 T_{0}$. One can notice that loss of symmetry has occurred. In reality the postbuckling state will be unstable for a while and recovers stability in cyclic manner. This is why characterising dynamic buckling presents an ambiguity for stiffened panels. Some authors consider that this final collapsing shape is the buckled state. One can notice however that the displacements are too high. Some authors have also defined the buckling state to be that for which the transverse displacement is three times the skin plate thickness [15]. Here, Budiansky-Roth criterion regarding violent oscillations was employed and an objective estimation for this issue was proposed. No one has indicated in fact how to determine in practice this state explicitly, and subjective interpretation of "large oscillation" or "large deformation under constant loading" as stated in the criterion can be made.

\section{Conclusions}

In this work, dynamic buckling of a longitudinally stiffened panel has been considered. The panel was subjected to short pulse in-plane axial compression applied on one transverse edge while the opposite edge was anchored and the three others were assigned symmetry about the transverse direction. Applying various dynamic loading having a half-sine pulse form, during the increasing part of the pulse and varying the pulse duration, has enabled to analyze dynamic buckling of this structure by using the Budiansky and Roth stability criterion. As this last is not fully explicit, a method for estimating the buckling state was proposed. Instability is assumed to occur for the state where a drop of reactions resultant magnitude attains half of its maximum value during oscillations. This has enabled to determine that the most severe dynamic buckling occurs when the pulse duration is close to two times the period of the first natural mode of vibrations. The dynamic load factor was found to be about $50 \%$, showing that dynamic buckling happening with these conditions of pulse period can be catastrophic for stiffened plates.

\section{References}

1. L. Ronning, A. Aalberg, P.K. Larsen P.K., Thin Walled Structures 48 (2010)

2. Z. Sadovsky, A.P. Teixeira, C. Guedes Soares, ThinWalled Structures 43 (2005)

3. J. Singer, J. Arbocz, T. Weller, Buckling experiments, experimental methods in buckling of thin walled structures (John Wiley \& Sons, 2002)

4. H.E. Lindberg, A.L. Florence, Dynamic Pulse Buckling: Theory and Experiment (M. Nijhoff, Boston, 1987)

5. C. Bisagni, Thin-Walled Structures 43(3) (2005)
6. G.J. Simitses, Dynamic stability of suddenly loaded structures (Springer-Verlag, New York, 1990)

7. B. Budiansky, R.S. Roth, Collected Papers on Instability of Shell Structures, NASA TN-D-1510 (1962)

8. B. Budiansky, Dynamic buckling of elastic structures: criteria and estimates (Pergamon Press, Oxford, 1967)

9. J. Wooseok, A.M. Waas, International Journal of Engineering Science 46 (2008)

10. T. Weller, H. Abramovich, R. Yaffe, Computers \& Structures 32 (1989)

11. H. Abramovich, A. Grunwald, Composites Structures 32 (1995)

12. Anonymous, Abaqus/Standard user's manual, ver. 6.10 (Hibbitt, Karlsson and Sorensen, 2010)

13. R.M.F. Paulo, F. Teixeira-Dias, R.A.F. Valente, Thin-Walled Structures 62 (2013)

14. I. Lillemäe, H. Remes, J. Romanoff, Thin-Walled Structures 72 (2013)

15. R.C. Batra, T.S. Geng, Smart Mater. Struct. 10 (2001) 